\title{
Design of Titanium Alloy Disc Based on Deformation Theory
}

\author{
Aiqin Lin \\ Department of Information Engineering, Liaoning Economic Management Cadre Institute, \\ Shenyang, 110122, China \\ Email: laq1206@163.com
}

Keywords: titanium alloy disc; finite element analysis; clamping way

Abstract. Through theoretical analysis and finite element analysis, the effect of the blunt force on the surface deformation is applied to the surface deformation of the fixture and the type surface. It obtains the most suitable for the titanium alloy plate clamping way.

\section{Introduction}

In the machining of the universal disk, the sensitive direction of force deformation of disk type workpiece is radial. For prevent severely deformed of workpiece when the clamp is clamped, directly affect the machining accuracy of the workpiece. It often uses to clamp the workpiece in a tightly clamped way to avoid processing error of workpiece precision. But it's easy to make fixtures, the inner ring and outer ring are tightly clamped by means of flat clamp. There is a gap between the shape of the disc and the fixture. It will analyze the effect of the gap on the processing accuracy of the titanium alloy disc.

\section{Modeling of titanium alloy disc surface bending deformation}

Because two surfaces of the titanium alloy membrane are quadratic equations, in order to simplify the model, the model of the workpiece is simplified to the beam. The most thin section of the selected film disc is not too bad for the deformation when the cutting force is used. Titanium diaphragm disc module is shown in Fig.1. The stress model the following assumptions:

(1) Because the thickness of the inner ring is much greater than the thickness of the plat, the outer ring and inner ring are assumed to be rigid during processing.

(2) It is assumed that the machining process of titanium alloy film disk is not transformed, the membrane disk is elastomer.

(3) The machining of titanium alloy disc is very small $\left(a_{p}=0.04 \mathrm{~mm}\right)$.The main part of the tooling and the workpiece is the blunt radius of the blade $\left(r_{\mathrm{n}}=0.04 \mathrm{~mm}\right)$.It is much smaller than the film plate $(30 \mathrm{~mm})$. So it can be assumed that the load is a point load, and the load is axial cutting force.

(4) At any point on the surface, the transfiguration is unchanged from the front and back.

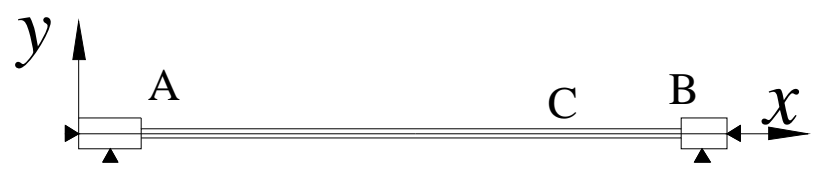

Fig. 1 Diagram of diaphragm disc 
According to the technical requirement of the film disc, it is considered the extreme condition of the deformation of the cutting force in the membrane plate. The thinnest section of the shape that affects the precision of the film disc shape is the most susceptible to deformation to the cutting force F. The beam's thickness is the thickness of the finishing process of the film disc for $0.946 \mathrm{~mm}$. The width of the beam is $1 \mathrm{~mm}$. The deformation of the film disk after the cutting force is shown in figure 2 .

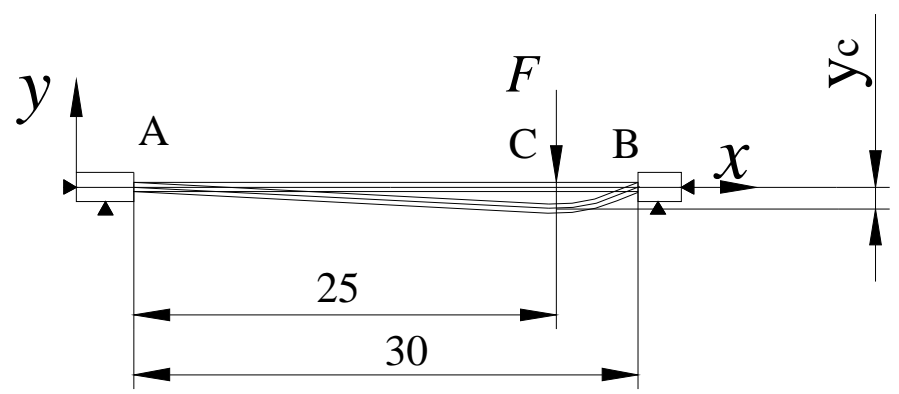

Fig. 2 Force diagram of diaphragm disc

\section{Bending deformation calculation of membrane plate surface}

Theoretical calculation condition of titanium alloy film disc: material Ti6Al4V, E=121 Gpa, $v=0.34$ 。 The minimum thickness of the disci is $0.946 \mathrm{~mm}$, width is $1 \mathrm{~mm}$ 。 $r_{\mathrm{n}}=0.04 \mathrm{~mm}$, $\mathrm{v}=100 \mathrm{~m} / \mathrm{min}, f=0.04 \mathrm{~mm} / \mathrm{r}, a_{p}=0.04 \mathrm{~mm}$. Use the ThirdWave. Cutting force perpendicular to the surface of the workpiece $F=5 N$.Request deformation of $\mathrm{C}$.

The moment of inertia of the rectangular beam: $I_{z}=\frac{b h^{3}}{12}$,

$$
\begin{aligned}
I_{z} & =\frac{1 \times 0.946^{3}}{12} \\
& =7.05 \times 10^{-2} \mathrm{~mm}^{4}
\end{aligned}
$$

The membrane disk is at point $\mathrm{C}$ :

$$
M_{C}=5 \mathrm{~N} \times 25 \times 10^{-3} \mathrm{~m}=0.125 \mathrm{Ngn}
$$

$\sigma_{t}=\frac{M_{C} y_{c}}{I_{z}}$ is known:

$$
y_{c}=\frac{\sigma I_{z}}{M_{c}}
$$

The yield strength of Ti6Al4V $\sigma=805 \mathrm{Mpa}$ :

$$
y_{c}=\frac{\sigma I_{z}}{M_{C}}=\frac{805 \times 10^{6} \mathrm{~Pa} \times 7.05 \times 10^{-2} \times\left(10^{-3}\right)^{4} \mathrm{~m}^{4}}{0.125 \mathrm{Ngn}}=0.454 \mathrm{~mm}
$$

The deformation quantity of disc on the shaft $0.454 \mathrm{~mm}$ is much greater than $0.025 \mathrm{~mm}$ film disk surface allows the deformation quantity. The results of the theoretical calculation show that the deformation of the clamp is beyond the value of the deformation of the workpiece. Before processing, the workpiece is too distorted, so the flat fixture is not suitable for the titanium alloy 
film disc.

\section{Tianium alloy diaphragm plate type tooling fixture of the axial clamping}

To fill the membrane type plate surface caused by the gap between the clamp and membrane on the surface of the disc type processing deformation, it develops titanium alloy membrane disk axial clamping fixture. The axial deformation of the axial surface of the workpiece is offset by the method of fully applying the fixture surface and the workpiece surface. It has a good effect in actual processing.

The processing technology of titanium alloy film disc is divided into rough machining, semi-finishing and finishing. The sides of the disc are not the same. As the process progresses, the type surface of the titanium alloy disc also changes. In order to ensure that the membrane plate and fixture face can be applied at different processing stage, different types of surface clamp are used at different stages of processing. Shape fixture of making the corresponding set is shown in fig.3.
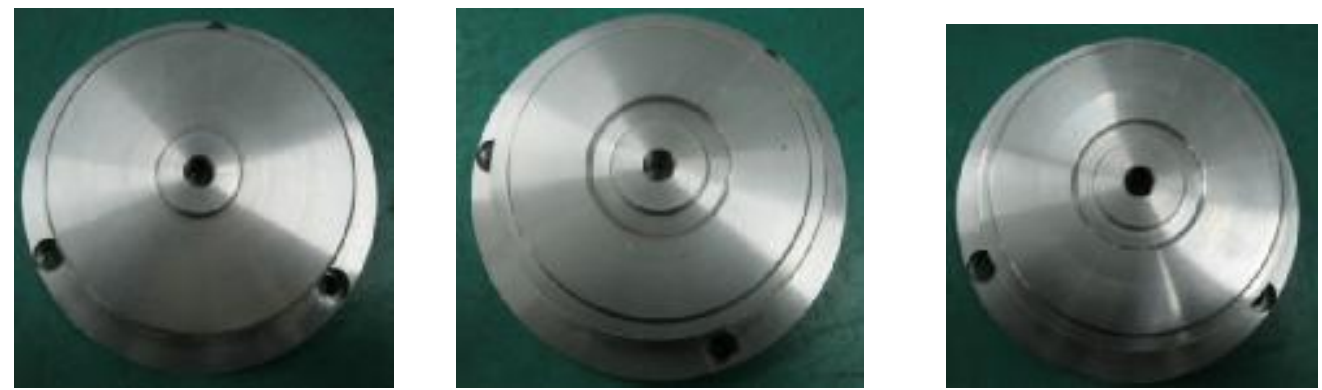

a) Rough machining fixture b) Semi-finished processing fixture c) Finish machining fixture

Fig.3 Processing complete sets of special fixtures of Titanium diaphragm disc

In order to ensure the accuracy between fixture replacement, and ensure the relative position between the workpiece and cutting tool, the jig design between three jaw chuck and the corresponding orientation check mouth and grooves. The material of the groove is 45 steel. In order to prevent the clamping of the clamp, the machine will not be removed in future processing, so as to guarantee the unity between the fixture and the machine tool. It connects to the position stop and fixture with bolts. The positioning accuracy of the diaphragm plate and the tool is used to ensure the accuracy of the alignment between the stop and the grooves. Before the test, the circle of the outside circle with the measuring fixture was less than $0.01 \mathrm{~mm}$. The processing precision of the film disk can be guaranteed. The positioning and positioning grooves of the titanium alloy diaphragm plate are shown in figure 4.

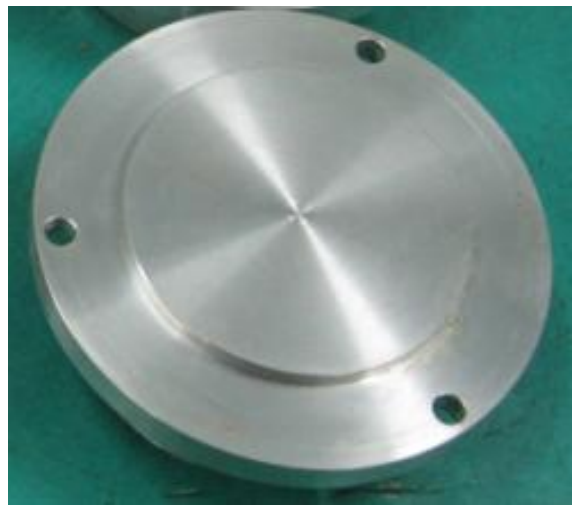

a) Positioning mouth

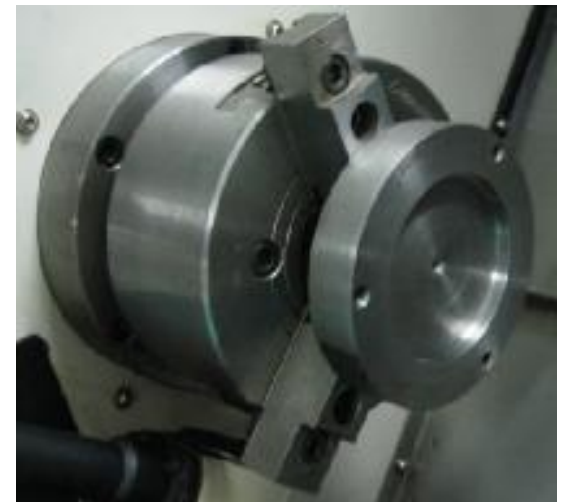

b) notch of special fixtures

Fig.4 Positioning mouth and notch of special fixtures 


\section{Conclusions}

Through tooling profile optimization design and the axial clamping fixture structure and cutter blade optimization results integration, developed a new type of aircraft engine titanium alloy plate membrane of complete sets of equipment and tooling. The processing deformation caused by different parts of the titanium alloy film plate is effectively reduced.

\section{References}

[1] Yanyan Song, Yueqing Song. Aviation research and application progress of titanium alloy [J]. Rare Metal,2006, 12: 850-856.

[2]Lili Han. Prediction and simulation research of the deformation of thin-walled parts processing [D].Harbin: the dissertation of Harbin University of Science and Technology, 2009.

[3]N. Fang, Q. Wu. A comparative study of the cutting forces in high speed machining of Ti6Al4V and Inconel718 with a round cutting edge tool[J]. Journal of Materials Processing Technology, 2009, 209(9): 4385-4389.

[4]Yihang Fan. Tool wear characteristics and cutting performance study of Efficient cutting of titanium alloy Ti6Al4V [D].Harbin: the dissertation of Harbin University of Science and Technology, 2011.

[5]Shucai Yang, Minli Zheng, Deqiang Zhang, Ning Liu and Yihang Fan. Influence of Cutting Parameters on Characteristics of Serrated Chip When High-Efficiency Cutting Ti6A14V[C]. Solid State Phenomena, 2011, 175: 278-283. (EI: 20113214209320). 